\title{
Particle Size Effect on TL Emission of ZnS Nanoparticles and Determination of Its Kinetic Parameters
}

\author{
L. Robindro Singh ${ }^{1,2}$ and S. Dorendrajit Singh ${ }^{3}$ \\ ${ }^{1}$ Department of Physics, Mizoram University, P U College, Aizawl 796 001, India \\ ${ }^{2}$ Department of Nanotechnology, North-Eastern Hill University, Shillong 793 002, India \\ ${ }^{3}$ Department of Physics, Manipur University, Manipur 795 003, Canchipur, India \\ Correspondence should be addressed to L. Robindro Singh, robindro001@yahoo.co.in \\ Received 17 March 2012; Accepted 3 May 2012 \\ Academic Editor: Leonard Deepak Francis
}

Copyright (๑) 2012 L. R. Singh and S. D. Singh. This is an open access article distributed under the Creative Commons Attribution License, which permits unrestricted use, distribution, and reproduction in any medium, provided the original work is properly cited.

\begin{abstract}
Nanoparticles have large surface area, and most of the ions are lying on its surface. Could these surface ions be contributed in thermoluminescence emission or enhanced nonradiative transition? In view of this, we have prepared small sizes of $\mathrm{ZnS}$ nanoparticles at low temperature and made two samples, one as-prepared (size $\sim 3 \mathrm{~nm}$ ) and the other heat-treated at $1073 \mathrm{~K}$ (size $\sim 32 \mathrm{~nm}$ ). Characterization of the samples shows that the prepared phosphors are pure. Thermoluminescence (TL) glow curves could not be recorded in both samples without irradiation. Even for higher dose of $\gamma$-radiation the as-prepared samples could not show TL signal, but $1073 \mathrm{~K}$ heat-treated sample shows the TL signal. This may be due to the fact that smaller particles have large surface area compared to bigger particles, the surface ions may produce the nonradiative transitions. The kinetic parameters of the TL glow curves are evaluated by the conventional methods and compared with curve fitting computerised glow curve deconvolution (CGCD) technique. The variations in both techniques are found only \pm 0.02 . The shape factor of all the glow curves $\sim 0.48$, and these TL glow curves could be fitted with order of kinetics 1.5.
\end{abstract}

\section{Introduction}

The semiconductor nanoparticles in the groups II-VI have been studied extensively due to their intriguing physical properties compared to their bulk counterparts [1-10]. Optical properties of such materials can be tuned due to quantum size effects which effectively lead to a size-dependent variation of band gap. Among the most studied II-VI semiconductors are $\mathrm{ZnO}, \mathrm{CdS}, \mathrm{ZnS}$, and so forth [11-15] for their wide range of luminescence emissions from ultraviolet to infrared regions. $\mathrm{ZnS}$ is a blue emitting direct band gap semiconductor of energy gap $3.6 \mathrm{eV}$ with low phonon energy suitable in display devices and lighting applications. Extensive studies on the luminescence emissions of $\mathrm{ZnS}$ nanoparticles doped with transition metals or lanthanide ions at different sizes, $\mathrm{pH}$, cappants, and so forth have been carried out since the past decades [16-22]. There are few reports on the study of thermoluminescence characteristics of these materials in nanosize; in view of this, the present paper is prepared to study their luminescence characteristics at different particle sizes.

Thermoluminescence (TL) continues to be an active area of research because of its immense contribution in the fields of personnel and environmental dosimetry, dating of archeological artifacts, sediments and study of defects in solids. Irradiation on many insulating and semiconducting materials creates sufficient defects to act as trap centers for electron and holes. Upon heating the materials containing large number of electrons and holes trapped in their respective trap centers, these electrons and holes are released and recombine with opposite charges resulting luminescence called TL (thermoluminescence). In the case of nanomaterials, the surfaceto-volume ratio is large resulting in larger concentrations of surface states. The surface states are responsible for the production of TL glow curves, that is why Chen et al. [23] record TL glow curves in $\mathrm{ZnS}$ nanoparticle prior to irradiation. They also observed that the TL intensity increases with the decrease of particle sizes and concluded that the surface 
ions on the nanoparticles were responsible for the production of TL glow curves. Crystalline sizes of the particles take important role in producting luminescence emission of the phosphors, many researchers are working in this regard on different phosphors [24-29]. Studying the luminescence characteristics of phosphors on particle sizes will be beneficial in lighting applications.

The present work studies the photoluminescence and thermoluminescence (TL) of as-prepared and $1073 \mathrm{~K}$ heattreated undoped (pure) ZnS nanoparticles. Kinetic parameters such as the activation energy $(E)$, order of kinetics $(b)$, and frequency factor $(s)$ of the glow peaks are determined by initial rise [30], curve fitting [31] and compared with computerised glow curve deconvolution method [32].

\section{Experimental Section}

2.1. Preparation. Quantum sizes of undoped (pure) $\mathrm{ZnS}$ nanoparticles were prepared by coprecipitation reaction at low temperature. In order to get small sizes of the particles, slow release of the reactants at controlled manner would be needed $[33,34]$. Ethylene glycol acts as the reaction medium as well as the capping ligand. The precursors used for the production of $\mathrm{Zn}^{2+}$ was $\mathrm{Zn}\left(\mathrm{CH}_{3} \mathrm{COO}\right)_{2} \cdot 2 \mathrm{H}_{2} \mathrm{O}(99.05 \%$, EMerck), and thiourea (AR) for the production of $\mathrm{S}^{2-} \cdot 1 \mathrm{gm}$ of $\mathrm{Zn}\left(\mathrm{CH}_{3} \mathrm{COO}\right)_{2} \cdot 2 \mathrm{H}_{2} \mathrm{O}$ was dissolved in $25 \mathrm{~mL}$ ethylene glycol; warming the solution, a clear solution could be observed. This solution contained $\mathrm{Zn}^{2+}$ ions. In this solution, $2.0 \mathrm{~g}$ of thiourea (AR) was simply added and warmed the mixture at $353 \mathrm{~K}$, the solution again became transparent. The reaction medium was kept in nitrogen atmosphere, in order to avoid oxidation during reaction. The whole mixture was heated linearly up to $403 \mathrm{~K}$. After half an hour, light yellowish-white colloidal precipitate could be observed, the reaction temperature maintained at this temperature and continues another two hours. The precipitates so obtained are extracted by centrifugation and washed several times in excess methanol (AR). Special care has been taken to avoid oxidation, the powder samples so obtained were dried at room temperature.

2.2. Characterization. X-ray diffraction studies were carried out using a Philips powder X-ray diffractometer (model $\mathrm{PW} 1071)$ with $\mathrm{Ni}$-filtered $\mathrm{Cu}-k_{\alpha}$ radiation. The lattice parameters were calculated from the least square fitting of the diffraction peaks. The average crystallite sizes were calculated from the diffraction line-width based on Scherrer relation: $d=0.9 \lambda / \beta \cos \theta$, where $\lambda$ is the wavelength of $\mathrm{X}$-rays, and $\beta$ is the half maximum line width. To record UV-visible spectra, EDX and AFM, the powder samples were dispersed in dimethyl formide (DMF), then multiple dipping were carried out on ultrasonically cleaned glass slides.

Photoluminescence measurements were carried out at room temperature with a resolution of $3 \mathrm{~nm}$, using a Hitachi Instrument (F-4500) having a $150 \mathrm{~W}$ Xe lamp as the excitation source. Powder samples $(5 \mathrm{mg})$ were mixed with methanol, spread over a quartz plate, dried at $373 \mathrm{~K}$, and mounted inside the sample chamber for photoluminescence

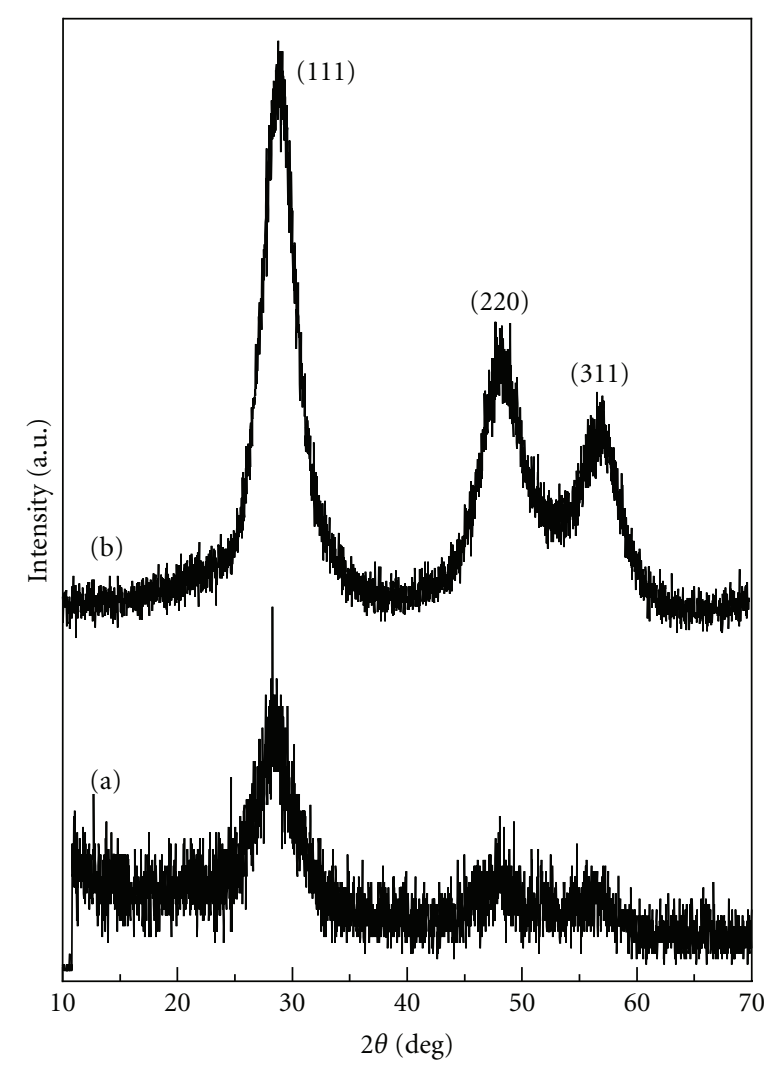

FIGURE 1: XRD pattern of (a) as-prepared and (b) $1073 \mathrm{~K}$ heattreated $\mathrm{ZnS}$ nanoparticles.

measurements. Thermoluminescence glow curves of the powder samples were recorded using TLD-1404 recorder.

\section{Results and Discussion}

3.1. X-Ray Diffraction (XRD) Study. Figure 1 shows the Xray diffraction patterns of (a) as-prepared and (b) $1073 \mathrm{~K}$ heat-treated ZnS nanoparticles and corresponds to cubic phase (JCPDS-77-2100) of space group F43m. No extra peaks of impurity could be detected even for higher heattreated sample within the resolution of limit diffractometer. The lattice parameter of the as-prepared $\mathrm{ZnS}$ nanoparticle is $a=5.359(1) \AA$ with unit cell volume $153.948(1) \AA^{3}$ and that of $1073 \mathrm{~K}$ heat-treated $\mathrm{ZnS}$ nanoparticle is $a=5.369(1)$ $\AA$ with unit cell volume $V=154.825(1) \AA^{3}$. The unit cell volume of heat-treated $\mathrm{ZnS}$ gives higher value compared to as-prepared $\mathrm{ZnS}$ nanoparticle. The increase of the unit cell volume with the increase of heat-treatment temperature may be due to the decrease in ionicity. The particle sizes calculated from Scherrer relation are found to be 3 and $32 \mathrm{~nm}$, respectively for as-prepared and $1073 \mathrm{~K}$ heat-treatment samples, respectively. The intensity of the diffraction peaks increases with the increase of the heat-treatment temperature showing an increase in the crystallinity of the samples with heattreatment.

3.2. EDX (Energy Dispersive X-ray) and AFM (Atomic Force Microscopy) Study. Figure 2 shows the EDX of as-prepared 


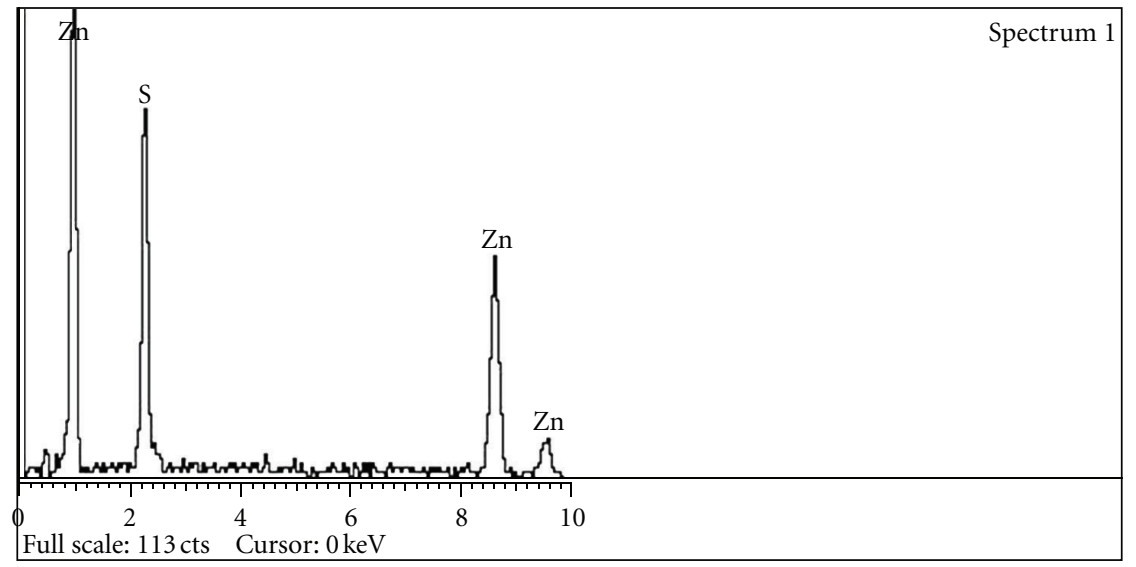

$(\mathrm{keV})$

FIgURE 2: EDX spectra of as-prepared ZnS nanoparticles.

$\mathrm{ZnS}$ nanoparticles. As-prepared sample gives the atomic $\% \mathrm{Zn}=54$ and $\mathrm{S}=46$, while the heat-treated sample gives atomic $\% \mathrm{Zn}=51$ and $\mathrm{S}=49$. Figure 3 shows the 2-dimensional AFM picture of (a) as-prepared and (b) $1073 \mathrm{~K}$ heattreated $\mathrm{ZnS}$ nanoparticles. The particles sizes determined by AFM are 12 and $87 \mathrm{~nm}$, respectively for the as-prepared and heat-treated $\mathrm{ZnS}$ nanocrystals. The particles in as-prepared samples are spherical in shape and agglomerated. Figure 3(c) is the log-normal distribution of as-prepared sample showing the particle size of this sample is $13 \mathrm{~nm}$. In the $1073 \mathrm{~K}$ heat-treated samples, the particles are spherical in shape and homogenous. It is obvious that the ethylene glycol can significantly resist agglomeration of nanoparticles.

\section{Luminescence Study}

4.1. TL Theory. Thermoluminescence is the thermally stimulated emission of light from insulators or semiconductors following previous absorption of energy from radiation. The electrons in the valance band gain energy due to exposure in radiation may come either to the conduction band or trapped in a metastable state as trapped electrons causing defect states in the material. On heating the phosphor, the trapped electrons in the metastable gain in energy then are released from the trapped level, if these electrons return to ground state with radiative recombination with the holes near the ground state, there is observation of thermoluminescence. When the recombination probability dominates the retrapping probability or retrapping probability is zero, Randall and Wilkins [35] had reported an expression for TL intensity of the first-order kinetics $(b=1)$ at a temperature $T$ as

$$
I(T)=n_{0} s \exp \left(-\frac{E}{k T}\right) \exp \left[-\frac{s}{\beta} \int_{T_{0}}^{T} \exp \left(-\frac{E}{k T^{\prime}}\right) d T^{\prime}\right]
$$

where $n_{0}$ is the initial concentration of trap electrons, $s$ the frequency factor, $E$ the activation energy, $k$ the Boltzmann constant, and $\beta$ the linear heating rate.
When the retrapping probability is the same as recombination probability then the expression for TL intensity of the second-order kinetics is given by [30]:

$$
I(T)=\frac{n_{0}^{2} s^{\prime} \exp (-E / k T)}{\left[1+\left(n_{0} s^{\prime} / \beta\right) \int_{T_{0}}^{T} \exp \left(-E / k T^{\prime}\right) d T^{\prime}\right]^{2}},
$$

where $s=n_{0} s^{\prime}$.

For the non-first-order kinetics $(b \neq 1)$, Gartia et al. [36] and Rasheedy [37] reported an expression for TL intensity as

$$
\begin{aligned}
I(T)= & N f^{b} s \exp \left(-\frac{E}{k T}\right) \\
& \times\left[1+(b-1) f^{b-1} \frac{s}{\beta} \int_{T_{0}}^{T} \exp \left(-\frac{E}{k T^{\prime}}\right) d T^{\prime}\right]^{-b /(b-1)},
\end{aligned}
$$

where $N$ is the electron traps and filling factor $f=n_{0} / N$.

The condition for maximum intensity for the glow curve obeying (3) is given by

$$
\begin{gathered}
1+(b-1) f^{b-1}\left(\frac{s}{\beta}\right) \int_{T_{0}}^{T_{m}} \exp \left(-\frac{E}{k T^{\prime}}\right) d T^{\prime} \\
=\frac{b s k T_{m}^{2}}{\beta E} f^{b-1} \exp \left(-\frac{E}{k T_{m}}\right) .
\end{gathered}
$$

The shape factor $\mu_{g}$, of the glow curve can also be determined from the relation [32]:

$$
\mu_{g}=\frac{\delta}{\omega}=\frac{\left(T_{2}-T_{m}\right)}{\left(T_{2}-T_{1}\right)}
$$

where $T_{m}$ is the peak temperature of the glow curve, and $T_{1}$, and $T_{2}$ are the temperatures at half of the maximum intensity on the rising and falling sides of the glow curves.

4.2. Photoluminescence and Thermoluminescence Study. Figure 4 shows the photoluminescence emissions of as-prepared and $1073 \mathrm{~K}$ heat-treated $\mathrm{ZnS}$ nanoparticles excited at 


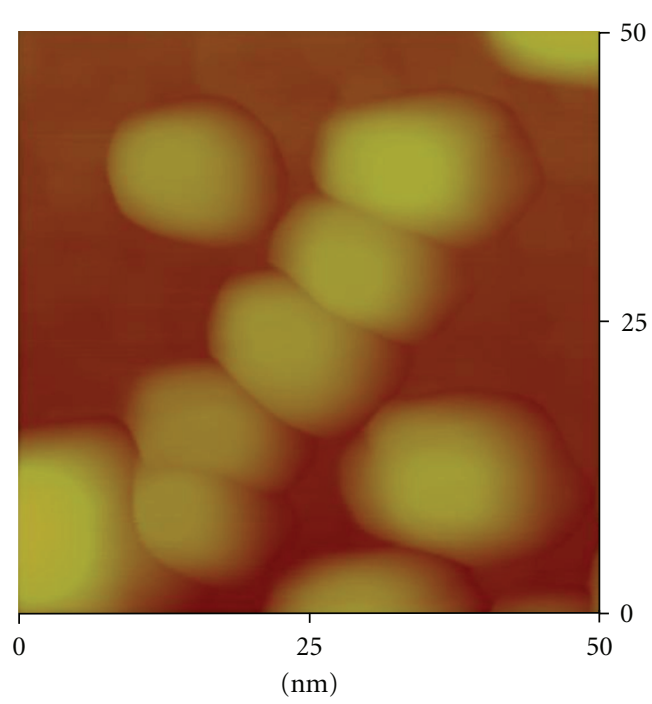

(a)

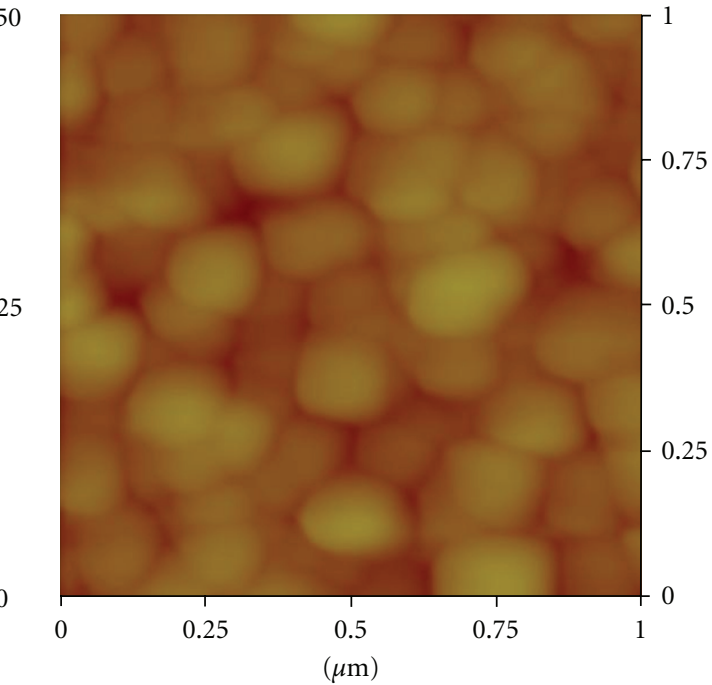

(b)

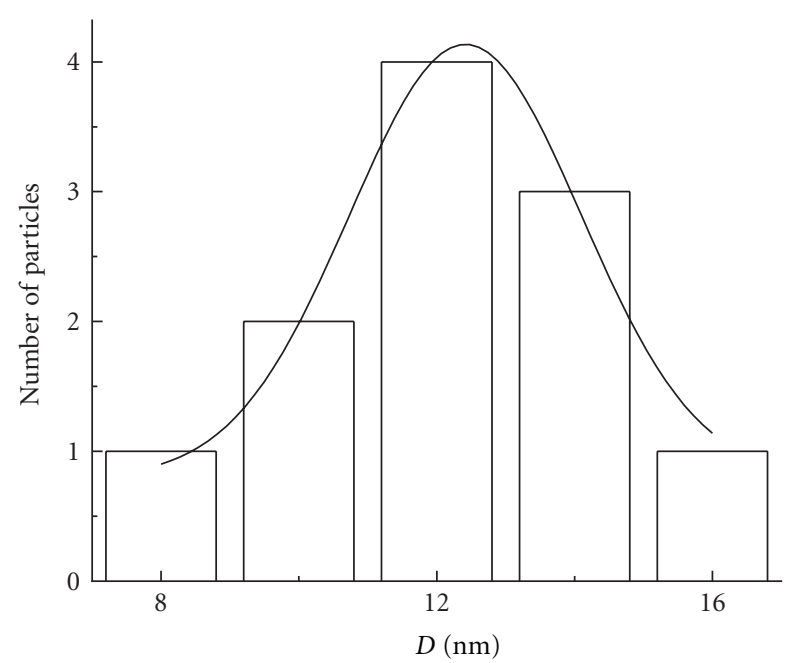

(c)

FiguRE 3: AFM picture of (a) as-prepared, (b) $1073 \mathrm{~K}$ heat-treated ZnS nanoparticles, and (c) log-normal distribution of the as-prepared nanoparticles showing that most of the particles are $13 \mathrm{~nm}$ in size.

$290 \mathrm{~nm}$. Strong blue emissions are observed, these emissions are the due to native intrinsic defects such as vacancies and interstitial form energy levels in ZnS, such as donor levels of $V_{\mathrm{S}}$ and $Z_{\mathrm{ni}}$ and acceptor level of $V_{\mathrm{zn}}$. The emission intensity increases with the increase of heat-treatment temperature, this observation may be due to increase in crystallinity of the particles which in turn reduces the iconicity and the surface effects of the nanoparticles.

Thermoluminescence (TL) glow curves were recorded for both as-prepared and heat-treated $\mathrm{ZnS}$ nanoparticles without irradiation, but no TL signal could be recorded. Both the as-prepared and heat-treated samples were irradiated with different doses of $\gamma$ radiation, but as-prepared sample does not show TL signals even for higher dose of $\gamma$ radiation. The $1073 \mathrm{~K}$ heat-treated $\mathrm{ZnS}$ nanoparticles were irradiated with 126 and 252 Gy of $\gamma$-rays, and TL glow curves could be recorded. Chen et al. [23] could get TL glow curves of
ZnS nanoparticles without irradiation. They observed that the TL intensity increases with the reduction of particle sizes. Their observations had open vehement argument for further understandings on the luminescence characteristics of small particles. They suggested that the surface ions on the nanoparticles took a vital role in the production of TL signals. But our observations are contrary to their findings. It is expected that that the surface ions on the surface of nanoparticles enhance the nonradiative transition that is why TL signal cannot be detected for as-prepared samples. It is well accepted that small particles have large surface area, and most of the ions lie on its surface. To confirm these observations, the as-prepared nanoparticles are heat-treated at $1073 \mathrm{~K}$ for one hour to get bigger particle sizes. Bigger nanoparticles have less number of surface ions and the non-radiative transition due to phonon-phonon interactions could be reduced extensively. Even this sample 
TABLE 1: Peak parameters of the TL glow curves irradiated with 126 and 252 Gy of $\gamma$-rays.

\begin{tabular}{lcccccc}
\hline Dose & $T_{m}(\mathrm{~K})$ & $I_{m}$ & $\tau$ & $\delta=T_{2}-T_{m}$ & $\omega=T_{2}-T_{1}$ & $\mu_{g}=\delta / \omega$ \\
\hline 126 & 374.0 & 66.3 & 43.5 & 50.5 & 101.0 & 0.50 \\
252 & 390.0 & 112.9 & 43.5 & 39.5 & 83.0 & 0.48 \\
\hline
\end{tabular}

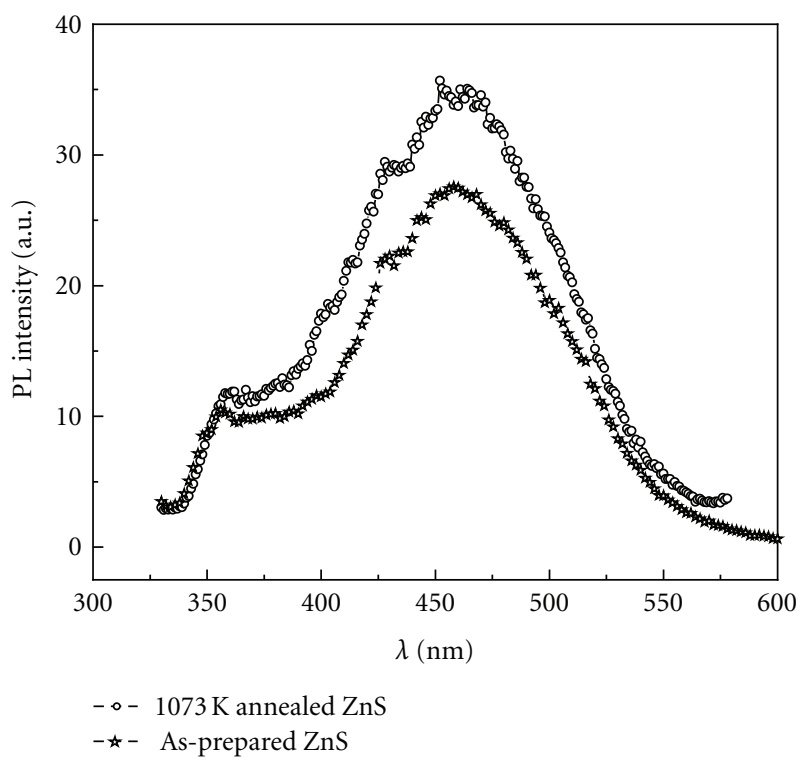

FIGURE 4: Photoluminescence emission spectra of as-prepared and $1073 \mathrm{~K}$ heat-treatment $\mathrm{ZnS}$ nanoparticles showing the native blue emission.

the TL signal could not be recorded prior to irradiation. Then the samples are again irradiated with $\gamma$ radiations, and TL signal could be recorded for different doses of radiations. With irradiation different trap centers could be developed, even though there are surface ions on the nanoparticles. The radiative transitions due to trap levels dominates over the non-radiative transitions due to surface ions. That is why, TL signals could be recorded in heat-treated and irradiated samples. The above observations revealed that the surface ions on the surface of the nanoparticles could not contribute to the luminescence emissions, but only the trap centers could give luminescence emissions. Figure 5 shows the TL glow curves of $1073 \mathrm{~K}$ heat-treated $\mathrm{ZnS}$ nanoparticle irradiated with 126 and 252 Gy of $\gamma$-rays with a linear heating rate of $1.73 \mathrm{~K} / \mathrm{s}$, and the peak temperatures of the glow curves are around 374 and $390 \mathrm{~K}$, respectively. The TL intensity of the glow curves varies with the different doses of $\gamma$-rays. The sample irradiated with higher dose of $\gamma$-ray has more intense in TL signal than the sample irradiated with lower $\gamma$ dose. The full widths $(\omega)$ of the glow curves are found to be $83 \mathrm{~K}$ and $101 \mathrm{~K}$, which are exceptionally large. In general, such a broad peak must contain at least two or more TL peaks. The shape factor $\mu_{g}$ is found to be 0.48 , which are the characteristics of the non-first-order kinetics model [30, 31]. Table 1 indicates the characteristic peak parameters of the recorded glow curves of Figure 7.

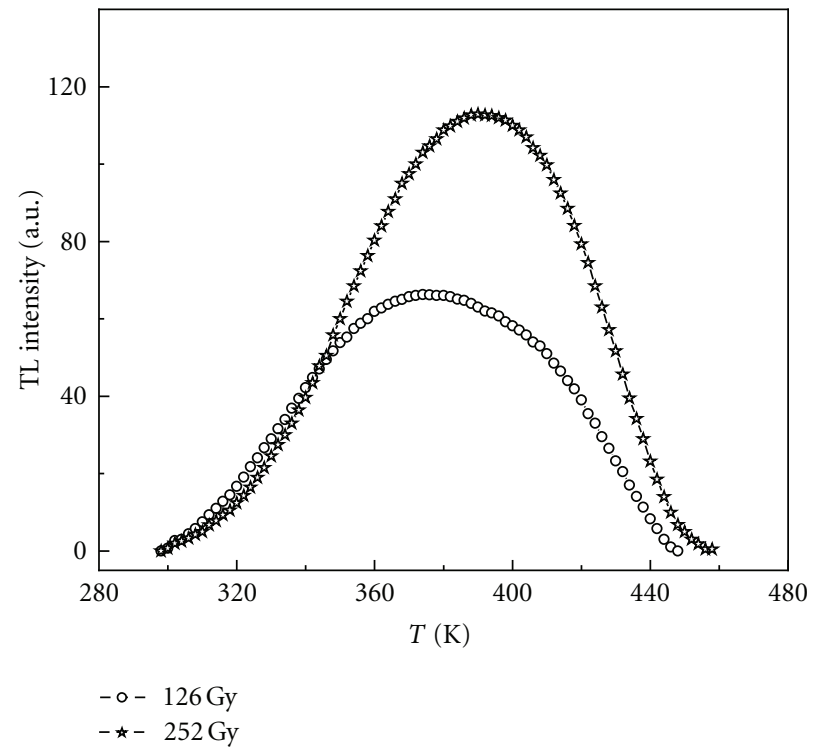

FIGURE 5: TL glow curves of nanoparticle $\mathrm{ZnS}$ irradiated with (a) $126 \mathrm{~Gy}$ and (b) $252 \mathrm{~Gy}$ of $\gamma$-rays with a linear heating rate $\beta=$ $1.73^{\circ} \mathrm{C} / \mathrm{s}$.

Figure 6 shows the TL glow curves of $1073 \mathrm{~K}$ heattreated $\mathrm{ZnS}$ nanoparticles irradiated with $126 \mathrm{~Gy}$ and $252 \mathrm{~Gy}$ of $\gamma$-rays after thermal cleaning up to $393 \mathrm{~K}$. The peak temperatures of the glow curves (126 Gy and $252 \mathrm{~Gy}$ ) are observed around $419 \mathrm{~K}$, and the full widths at half maximum $(\omega)$ of the glow curves become 41 and $47 \mathrm{~K}$, respectively. The shape factors of the glow curves are still at 0.48. The 252 Gy irradiated sample has been thermally cleaned to different temperatures $373,393,413$, and $443 \mathrm{~K}$, and then the glow curves are recorded. The peak temperatures of the recorded glow curves are 409.15, 415.82, 423.38, and $434.2 \mathrm{~K}$, respectively as shown in Figure 7. The full width $(\omega)$ becomes narrower as compared to the glow curves before thermal cleaning. The value of the shape factor of the glow curves is at $\sim 0.48$, which is the characteristic of the non-first-order kinetics. Physically, it signifies that during heating parts of the electrons released from the traps are recombining with the holes in the hole centers resulting TL, and the remaining part of electrons are retrapped back to the electron traps. If significant numbers of electrons are retrapped back to the earlier electron trap centres such that retrapping probability is equal to recombination probability, the shape factor should be nearly 0.52 . Table 2 describes the peak parameters of the glow curves, which are thermally cleaned at temperatures $373,393,413$, and $443 \mathrm{~K}$. It is clearly observed that all the peaks are non-first-order kinetics. 
TABLe 2: Peak parameters of $\gamma$-irradiated TL glow curves of $1073 \mathrm{~K}$ heat-treatment $\mathrm{ZnS}$ nanoparticles recorded with different thermal cleaning $(T c)$.

\begin{tabular}{lcccccc}
\hline$T c(\mathrm{~K})$ & $T_{m}(\mathrm{~K})$ & $I_{m}$ & $\tau$ & $\delta=T_{2}-T_{m}$ & $\omega=T_{2}-T_{1}$ & $\mu_{g}=\delta / \omega$ \\
\hline 373 & 409.15 & 99.2 & 38.4 & 30.0 & 29.8 & 60.8 \\
393 & 415.82 & 89.6 & 36.1 & 24.6 & 51.4 & 0.47 \\
413 & 423.38 & 63 & 31.4 & 22.35 & 44.7 & 0.48 \\
443 & 434.20 & 16.6 & 16.1 & 0.50 \\
\hline
\end{tabular}

TABLE 3: Comparison of activation energies calculated from CGCD and peak shape method of the $\gamma$-irradiated ZnS nanoparticles.

\begin{tabular}{lccccc}
\hline Dose $(\mathrm{Gy})$ & $T_{m}(\mathrm{~K})$ & $E c(\mathrm{eV})$ & $E_{\delta}$ & $b$ & $s\left(\mathrm{sec}^{-1}\right)$ \\
\hline \multirow{3}{*}{126} & 396.92 & 0.67 & 0.634 & 1.5 & $5.9 \times 10^{7}$ \\
& 427.16 & 0.95 & 0.891 & 1.5 & $2.3 \times 10^{10}$ \\
252 & 391.25 & 0.70 & 0.732 & 1.5 & $1.5 \times 10^{7}$ \\
\hline
\end{tabular}

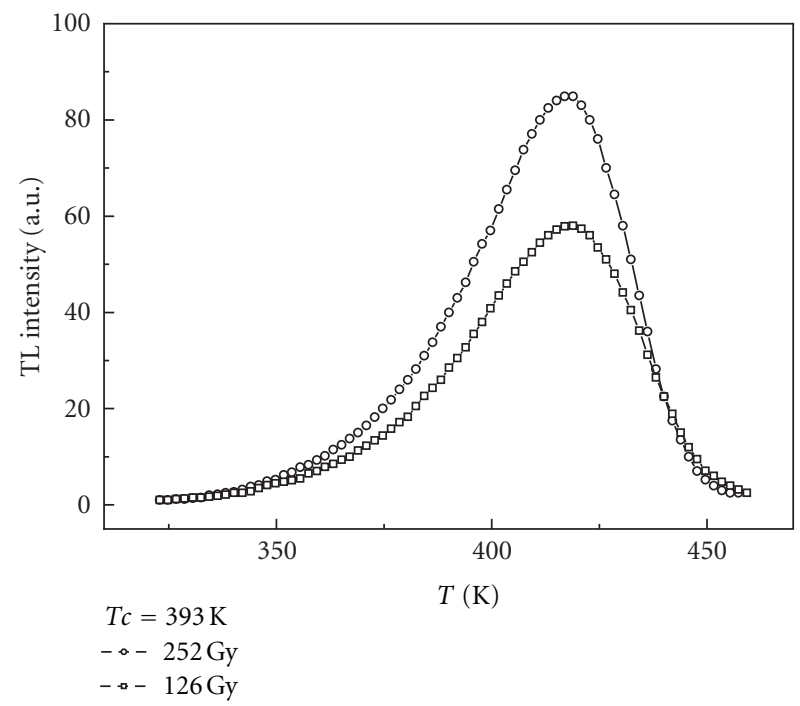

FIgURE 6: TL glow curves of nanoparticle $\mathrm{ZnS}$ irradiated with (a) $126 \mathrm{~Gy}$ and (b) $252 \mathrm{~Gy}$ of $\gamma$-rays recorded after thermal cleaning up to $393 \mathrm{~K}\left(\beta=1.73^{\circ} \mathrm{C} / \mathrm{s}\right)$.

To determine kinetic parameters such as activation energy $(E)$, order of kinetics $(b)$, and frequency factor $(s)$ of the observed TL glow curves, we have used the conventional methods such as initial rise (IR) [30], peak shape method [31], and CGCD technique [32, 36, 38]. A series of initial rise (IR) thermoluminescence curves are recorded for the sample irradiated with 252 Gy of $\gamma$-ray by keeping the voltage across the photomultiplier tube to a high value such that TL intensity becomes large. The magnitude of the slope of In $(I)$ versus $1 / T$ gives $E / k$, and consequently $E$ (activation energy) can be evaluated. The mean value of activation energy by using IR method is $0.78 \mathrm{eV}$. It is to be noted that the IR method to determine $E$ is independent of the order of kinetics. Again the activation energy is evaluated using the peak shape formula of Gartia et al. [39]. The comparison between trapping parameters of $\mathrm{ZnS}$ nanoparticles evaluated by peak shape method and CGCD is shown in Table 3.

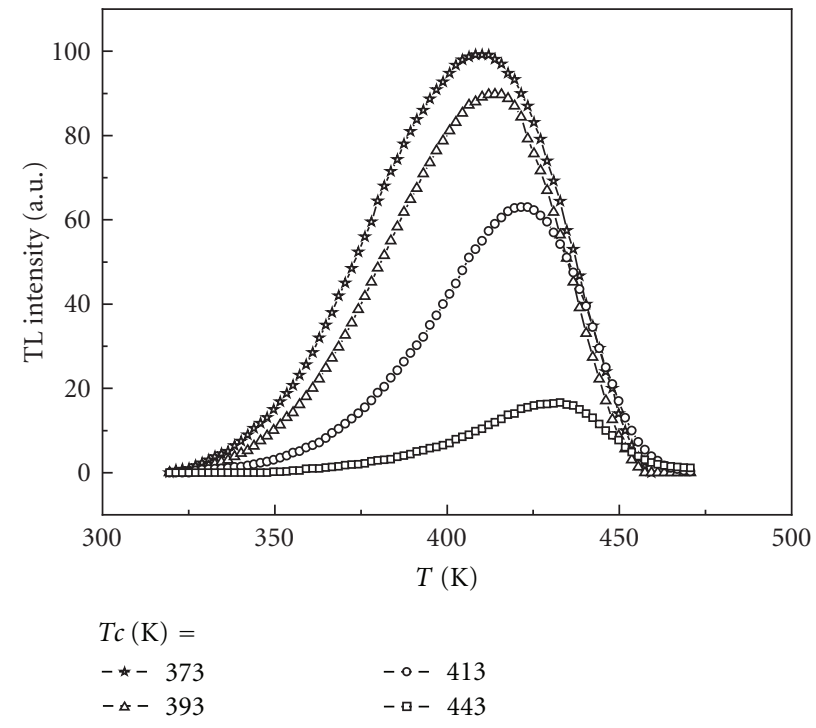

Figure 7: TL glow curves of ZnS irradiated with $252 \mathrm{~Gy}$ of $\gamma$-ray recorded after thermal cleaning at (a) $373 \mathrm{~K}$, (b) $393 \mathrm{~K}$, (c) $413 \mathrm{~K}$, and (d) $443 \mathrm{~K}$ with linear heating rate $1.73^{\circ} \mathrm{C} / \mathrm{s}$.

Figure 8 shows curve fitting of the glow curve of $\mathrm{ZnS}$ irradiated with 126 Gy of $\gamma$-rays after thermally cleaned up to $393 \mathrm{~K}$. It can be fitted with two peaks with peak temperatures 396.92 and $427.16 \mathrm{~K}$. The thermal activation energies of the deconvoluted curves are 0.7 and $1.0 \mathrm{eV}$, and frequency factors of the deconvoluted curves are $5.9 \times 10^{7}$ and $2.3 \times 10^{10} \mathrm{~s}^{-1}$. Both the deconvoluted peaks are non-first-order of kinetics $b=1.5$. Similar pattern can be observed for higher $T c$ samples. Similar patterns are also observed for 252 Gy $\gamma$-ray irradiated sample. The deconvoluted glow curves have peak temperatures at 391.25 and $421.5 \mathrm{~K}$, with order of kinetics $b=1.5$. Table 3 indicates the activation energies calculated from CGCD and shape factor technique of $\mathrm{ZnS}$ nanoparticle irradiated with 126 and $252 \mathrm{~Gy}$ of $\gamma$-rays. The activation energy $(E c)$ determined from CGCD and shape factor $\left(E_{\delta}\right)$ is almost consistent, the difference is \pm 0.02 . 


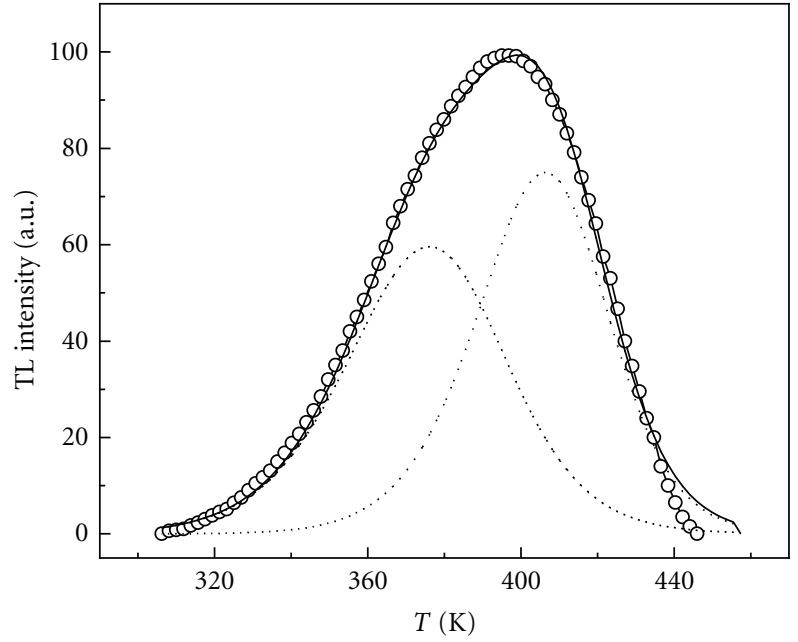

Figure 8: Computerised glow curve deconvolution of $\mathrm{ZnS}$ nanoparticles irradiated with 252 Gy of $\gamma$ after thermal cleaning of $393 \mathrm{~K}$ with linear heating rate of $1.73^{\circ} \mathrm{C} / \mathrm{s}$, (ooo) Expt. Glow curve (-) sum of deconvoluted curves and $(\cdots)$ deconvoluted curves.

\section{Conclusion}

Small sizes of $\mathrm{ZnS}$ nanoparticles could be prepared successfully at low temperature, it is evident that ethylene glycol can significantly resist agglomeration of the nanoparticles even heated at higher temperatures. As-prepared and $1073 \mathrm{~K}$ heat-treatment samples could not show TL signal without irradiation, reduction of surface ions on the nanoparticles might be responsible for the production of TL signals. The order of kinetics as evaluated by shape factor (non-first-order of kinetics) has the same pattern as evaluated by curve fitting technique. Peak shape method could be suitable to determine activation energy of the glow curves. Order of kinetics of the glow curves has the same value on order of kinetics $b=1.5$ even for different doses of irradiation. The calculated value of activation energy using peak shape formula is in agreement with that of fitted value.

\section{Acknowledgments}

The authors owe R. S. Ningthoujam, scientific officer E. Chemistry Division, BARC, Mumbai for providing the facilities of characterizations and luminescence measurements. L. R. Singh thanks Dr. T. D. Singh, Swambung College, Imphal for his constant encouragement and suggestions while preparing this work. L. R. Singh thanks to DST-New Delhi for financial assistance under the scheme of DST Fast Tract Scheme for Young Scientist during execution of the present work.

\section{References}

[1] A. Balandin, K. L. Wang, N. Kouklin, and S. Bandyopadhyay, "Raman spectroscopy of electrochemically self-assembled CdS quantum dots," Applied Physics Letters, vol. 76, no. 2, pp. 137$140,2000$.
[2] R. N. Bhargava, D. Gallagher, X. Hong, and A. Nurmikko, "Optical properties of manganese-doped nanocrystals of ZnS," Physical Review Letters, vol. 72, no. 3, pp. 416-419, 1994.

[3] L. E. Brus, "Zero dimensional "excitons" in semiconductor clusters IEEE," Journal of Quantum Electronics, vol. 22, no. 9, pp. 1909-1926, 1986.

[4] N. Taghavinia and T. Yao, "ZnS nanocrystals embedded in $\mathrm{SiO}_{2}$ matrix," Physica E, vol. 21, no. 1, pp. 96-102, 2004.

[5] M. J. Natan, J. W. Thackeray, and M. S. Wrighton, "Interaction of thiols with n-type cadmium sulfide and n-type cadmium selenide in aqueous solutions: adsorption of thiolate anion and efficient photoelectrochemical oxidation to disulfides," Journal of Physical Chemistry, vol. 90, no. 17, pp. 4089-4098, 1986.

[6] M. Ihara, T. Igarashi, T. Kusunoki, and K. Ohno, "Cathodoluminescence and photoluminescence of nanocrystal phosphors," Journal of the Electrochemical Society, vol. 149, no. 3, pp. H72-H75, 2002.

[7] A. A. Bol and A. Meijerink, "Long-lived $\mathrm{Mn}^{2+}$ emission in nanocrystalline $\mathrm{ZnS}: \mathrm{Mn}^{2+}$," Physical Review B, vol. 58, no. 24, pp. R15997-R16000, 1998.

[8] W. L. Wilson, P. F. Szajowski, and L. E. Brus, "Quantum confinement in size-selected, surface-oxidized silicon nanocrystals," Science, vol. 262, no. 5137, pp. 1242-1244, 1993.

[9] R. S. Ningthoujam, V. Sudarsan, S. V. Godbole, L. Kienle, S. K. Kulshreshtha, and A. K. Tyagi, "Sn $\mathrm{O}_{2}: \mathrm{Eu}^{3+}$ nanoparticles dispersed in $\mathrm{TiO}_{2}$ matrix: improved energy transfer between semiconductor host and $\mathrm{Eu}^{3+}$ ions for the low temperature synthesized samples," Applied Physics Letters, vol. 90, no. 17, Article ID 173113, 3 pages, 2007.

[10] A. D. Yoffe, "Low-dimensional systems: Quantum size effects and electronic properties of semiconductor microcrystallites (zero-dimensional systems) and some quasi-two-dimensional systems," Advances in Physics, vol. 42, no. 2, pp. 173-266, 1993.

[11] A. P. Alivisatos, "Semiconductor nanocrystals," MRS Bulletin, vol. 20, no. 8, pp. 23-32, 1995.

[12] C. C. Yang, S. Y. Cheng, H. Y. Lee, and S. Y. Chen, "Effects of phase transformation on photoluminescence behavior of $\mathrm{ZnO}: \mathrm{Eu}$ prepared in different solvents," Ceramics International, vol. 32, no. 1, pp. 37-41, 2006.

[13] M. Lomascolo, A. Cretì, G. Leo, L. Vasanelli, and L. Manna, "Exciton relaxation processes in colloidal core/shell $\mathrm{ZnSe} / \mathrm{ZnS}$ nanocrystals," Applied Physics Letters, vol. 82, no. 3, article 418, 3 pages, 2003.

[14] N. Karar, F. Singh, B. R. Mehata et al., "Structure and Photoluminescence studies on ZnS:Mn nanopaticles," Journal of Applied Physics, vol. 95, no. 2, pp. 656-661, 2004.

[15] K. K. Nanda and S. N. Sahu, "Photoluminescence of CdS nanocrystals: effect of ageing," Solid State Communications, vol. 111, no. 12, pp. 671-674, 1999.

[16] B. A. Smith, J. Z. Zhang, A. Joly, and J. Liu, "Luminescence decay kinetics of $\mathrm{Mn}^{2+}$-doped $\mathrm{ZnS}$ nanoclusters grown in reverse micelles," Physical Review B, vol. 62, no. 3, pp. 20212028, 2000.

[17] I. V. Kityk, M. Makowska-Janusik, J. Ebothé, A. El Hichou, B. El Idrissi, and M. Addou, "Photoinduced non-linear optical effects in the $\mathrm{ZnS}-\mathrm{Al}$, In-Sn doped film-glass nanometer-sized interfaces," Applied Surface Science, vol. 202, no. 1-2, pp. 2432, 2002.

[18] A. A. Bol, J. Ferwerda, J. A. Bergwerff, and A. Meijerink, "Luminescence of nanocrystalline $\mathrm{ZnS}: \mathrm{Cu}^{2+}$," Journal of Luminescence, vol. 99, no. 4, pp. 325-334, 2002.

[19] L. Xu, X. Huang, H. Huang, H. Chen, J. Xu, and K. Chen, "Surface modification and enhancement of luminescence due to 
quantum effects in coated $\mathrm{CdSe} / \mathrm{CuSe}$ semiconductor nanocrystals," Japanese Journal of Applied Physics, Part 1, vol. 37, no. 6, pp. 3491-3494, 1998.

[20] S. L. Cumberland, K. M. Hanif, A. Javier et al., "Inorganic clusters as single-source precursors for preparation of CdSe, $\mathrm{ZnSe}$, and CdSe/ZnS nanomaterials," Chemistry of Materials, vol. 14, no. 4, pp. 1576-1584, 2002.

[21] H. Benisty, C. M. Sotomayor-Torrès, and C. Weisbuch, "Intrinsic mechanism for the poor luminescence properties of quantum-box systems," Physical Review B, vol. 44, no. 19, pp. 10945-10948, 1991.

[22] J. F. Suyver, R. Bakker, A. Meijerink, J. J. Kelly et al., "Photoelectrochemical characterization of nanocrystalline $\mathrm{ZnS}: \mathrm{Mn}^{2+}$ layer," Physica Status Solidi, vol. 224, no. 1, pp. 307-312, 2001.

[23] W. Chen, Z. Wang, Z. Lin, and L. Lin, "Absorption and luminescence of the surface states in ZnS nanoparticles," Journal of Applied Physics, vol. 82, no. 6, pp. 3111-3115, 1997.

[24] S. J. Dhoble, P. M. Bhujbal, N. S. Dhoble, and S. V. Moharil, "Lyoluminescence, thermoluminescence and photodecomposition in microcrystalline powder of $\mathrm{KCl}, \mathrm{KBr}, \mathrm{KI}$ and $\mathrm{KI}: \mathrm{KNO}_{3}$ crystals," Nuclear Instruments and Methods in Physics Research, Section B, vol. 192, no. 3, pp. 280-290, 2002.

[25] R. Sharma, D. P. Bisen, S. J. Dhoble, N. Brahme, and B. P. Chandra, "Mechanoluminescence and thermoluminescence of Mn doped ZnS nanocrystals," Journal of Luminescence, vol. 131, no. 10, pp. 2089-2092, 2011.

[26] A. Jagannatha Reddy, M. K. Kokila, H. Nagabhushana et al., "Structural, EPR, photo and thermoluminescence properties of ZnO:Fe nanoparticles," Spectrochimica Acta-Part A, vol. 133, no. 2-3, pp. 876-883, 2011.

[27] A. N. Yazici, M. Öztaş, and M. Bedir, "The thermoluminescence properties of copper doped $\mathrm{ZnS}$ nanophosphor," Optical Materials, vol. 29, no. 8, pp. 1091-1096, 2007.

[28] A. B. de Carvalho Jr., P. L. Guzzob, H. L. Sullasic, H. J. Khouryd et al., "Effect of particle size in the TL response of natural quartz sensitized by high dose of gamma radiation and heattreatments," Materials Research, vol. 13, no. 2, pp. 265-271, 2010.

[29] A. N. Yazici, M. Öztaş, and M. Bedir, "Effect of sample producing conditions on the thermoluminescence properties of ZnS thin films developed by spray pyrolysis method," Journal of Luminescence, vol. 104, no. 1-2, pp. 115-122, 2003.

[30] G. F. J. Garlick and A. F. Gibson, "The electron trap mechanism of luminescence in sulphide and silicate phosphors," Proceedings of the Physical Society, vol. 60, no. 6, pp. 574-590, 1948.

[31] S. Mahajna and Y. S. Horowitz, "The unified interaction model applied to the gamma ray induced supralinearity and sensitization of peak 5 in LiF:Mg,Ti (TLD-100)," Journal of Physics D, vol. 30, no. 18, pp. 2603-2619, 1997.

[32] R. K. Gartia, S. J. Singh, T. S. C. Singh, and P. S. Mazumdar, "On the peak shape method for the determination of activation energy in TSL and TSC," Journal of Physics D, vol. 26, no. 4, pp. 694-696, 1993.

[33] L. R. Singh, R. S. Ningthoujam, and S. D. Singh, "Tuning of ultra-violet to green emission by choosing suitable excitation wavelength in $\mathrm{ZnO}$ : quantum dot, nanocrystals and bulk," Journal of Alloys and Compounds, vol. 487, no. 1-2, pp. 466471, 2009.

[34] L. Robindro Singh, R. S. Ningthoujam, V. Sudarsan et al., "Luminescence study on $\mathrm{Eu}^{3+}$ doped $\mathrm{Y}_{2} \mathrm{O}_{3}$ nanoparticles: particle size, concentration and core-shell formation effects," Nanotechnology, vol. 19, no. 5, Article ID 055201, 2008.
[35] J. T. Randall and M. H. F. Wilkins, "The phosphorescence of Various solids,” MHF, vol. 184, no. 999, pp. 347-367, 1945.

[36] R. G. Gartia, S. D. Singh, and P. S. Mazumdar, "On the glow curve shape and plateau test: the case of non-first-order kinetics," Physica Status Solidi, vol. 138, no. 1, pp. 319-326, 1993.

[37] M. S. Rasheedy, "On the general-order kinetics of the thermoluminescence glow peak," Journal of Physics, vol. 5, no. 5, pp. 633-636, 1993.

[38] R. Chen and Y. Kirsh, Analysis of Thermally Stimulated Process, Pergamon, Oxford, UK, 1981.

[39] R. K. Gartia, S. J. Singh, and P. S. Mazumdar, "Determination of the activation energy of thermally stimulated luminescence peaks obeying general-order kinetics," Physica Status Solidi (A), vol. 114, no. 1, pp. 407-411, 1989. 

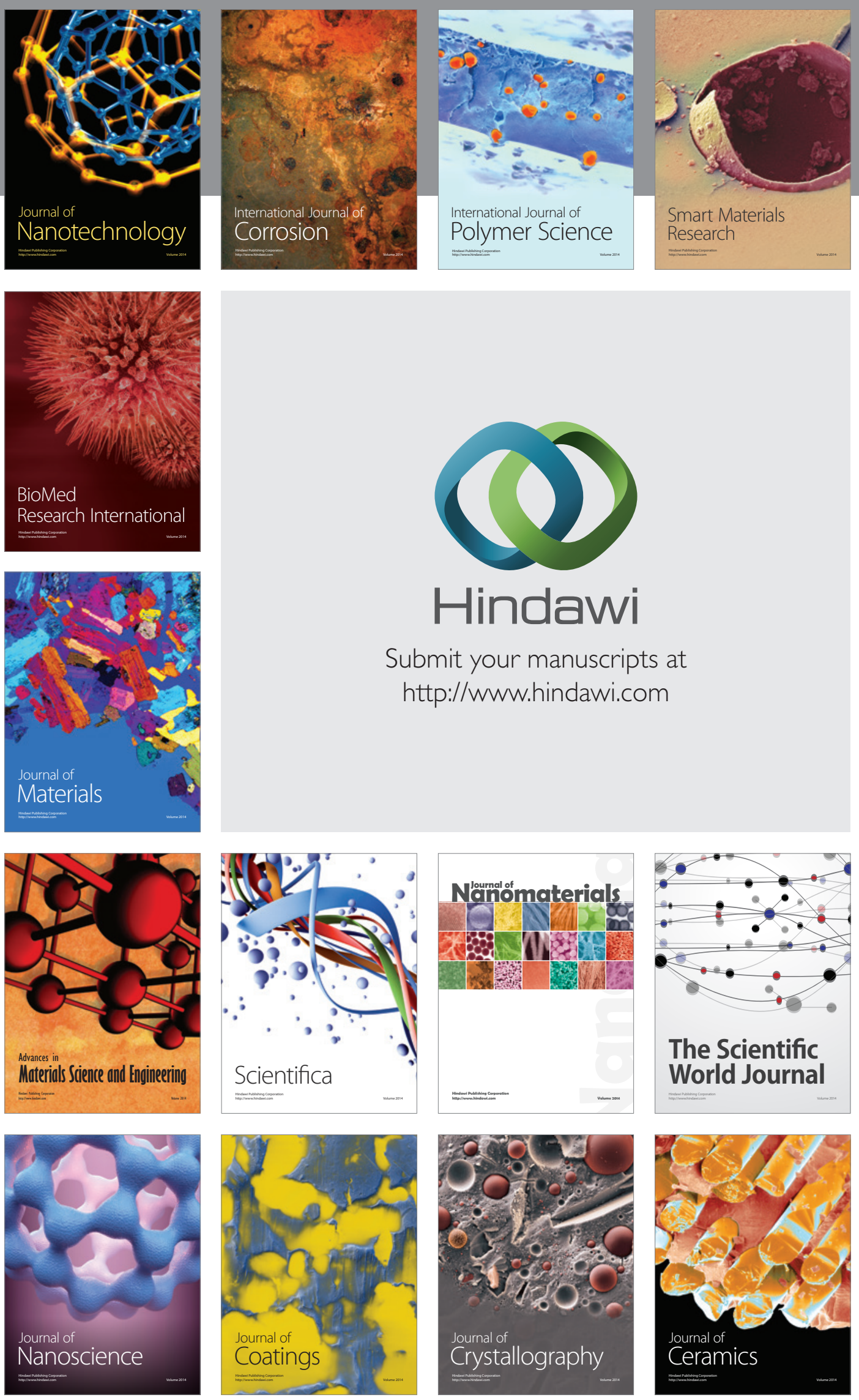

The Scientific World Journal

Submit your manuscripts at

http://www.hindawi.com

\section{World Journal}

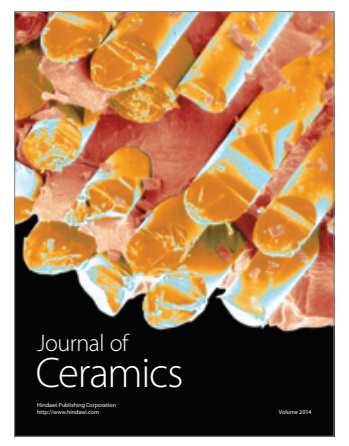

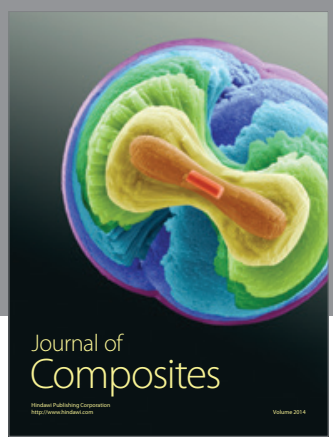
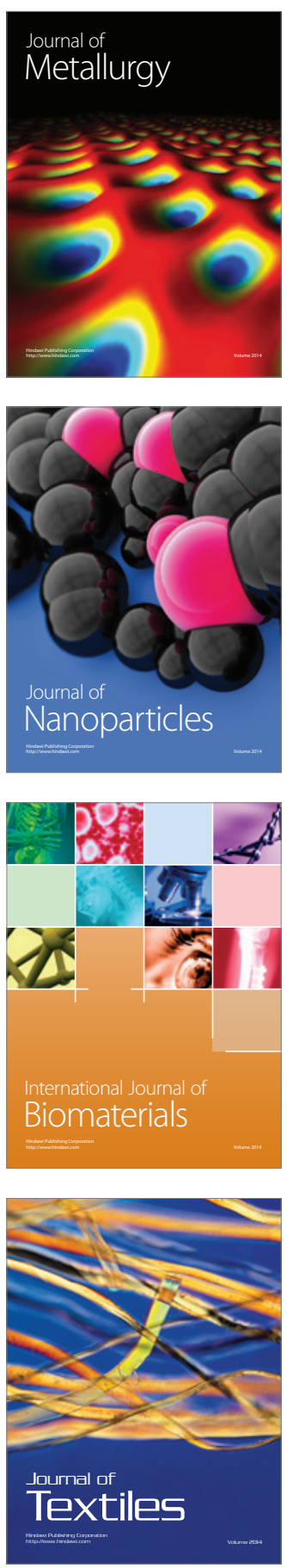